\title{
AUTOEFICACIA PERCIBIDA EN UNIVERSITARIOS. ESTUDIO DESCRIPTIVO
}

\author{
PERCEIVED SELF-EFFICACY \\ IN COLLEGE. \\ DESCRIPTIVE STUDY
}

Recibido 28 de octubre 2016 Aceptado diciembre 2016

Correspondencia: Escuela Nacional de Enfermería y Obstetricia jes_naj@hotmail.com

\section{Autores:}

Jesús Nájera Vuelvas

Pasante en Servicio Social. ENEO-UNAM

Ana Laura Pacheco Arce

Profesora de T.C. SUAYED. ENEO-UNAM

Cristina Rodríguez Becerra

Profesora de T.C. SUAYED. ENEO-UNAM

Irma Isabel Téllez Ortiz

Profesora de T.C. SUAYED. ENEO-UNAM

Teresa Sánchez Estrada

Profesora de Asignatura SUAyED ENEO-UNAM
Palabras clave: Autoellcacia, Salud, Universitarios, Nuevo ingreso, Enfermería.

Key words: Self-efficacy, Health, Universitaries, Incoming students, Nursing, Academic performance. 
RESUMEN

Problema: La autoeficacia percibida en alumnos de enfermería de nuevo ingreso.

Objetivos: Identificar mediante la escala de Ralph Schwarzer y Judith Baessler; los niveles de autoeficacia percibida para establecer una intervención que permita al participante ser resolutivo en la práctica clínica y en su vida personal.

Material y método: Diseño: Transversal, descriptivo, observacional, con una medición en una población. Universo. Alumnos de la licenciatura en enfermería de la ENEO-UNAM. Alumnos de nuevo ingreso. Se entrevistó a 342 Unidades de observación que constituyen el total de alumnos de nuevo ingreso. Criterios de selección de la muestra. Inclusión: Todos los alumnos que contestaron el formulario el día y la hora de su aplicación. Tratamiento estadístico de datos: Recolección: Encuesta, mediante entrevista directa instrumentada con formulario auło aplicable. Instrumento: Se utilizó la escala de autoeficacia Schwrzer - Baessler (1996) que califica con un alfa de Crombach de 0.86. Se procesaron con SPSS versión 23.0. El análisis es porcentual y se presenta en cuadros y graficas simples.

Resultados: El 20.2\% y $12.3 \%$ respondió cierto e incorrecto que pueden encontrar la manera de obtener lo que quieren a pesar de alguna oposición. El 48.5\% acierta a que le es fácil persistir en lo que se propone parar lograr sus metas. El 67.5\% dice que es cierto que si se esfuerza lo suficiente puede resolver problemas difíciles. Solo el 40.4\% dice que puede manejar acontecimientos inesperados eficazmente.

Palabras clave: Autoeficacia, Salud, Universitarios, Nuevo ingreso, Enfermería, Rendimiento académico.

\section{ABSTRACT}

Problem: Perceived self-efficacy in nursing students Incoming students admitted.

Objectives: To identify the scale by Schwarzer \& Baessler levels of perceived self-efficacy to establish an intervention that allows the participant to be operative in clinical practice and in his personal life.

Material and method: Design: Cross-sectional descriptive with a measurement in a population. Universe. Students of the pregraduate in nursing from the ENEO-UNAM. Incoming students. We interviewed 342 observation units that make up the total of new students. Criteria for selection of the sample. Inclusion: All students who answered the form on the day and time of your application. Exclusion: It's not excluded nor any case was removed. Statistical data processing: Collection: Survey by direct interview instrumented with applicable questionnaire. Instrument. self-efficacy scale Schwarzer \& Baessler (1996) qualifies with a Cronbach's alpha of 0.92 was used. They were processed with SPSS version 23.0. The analysis is presented in percentage and simple charts and graphs.

Results: $20.2 \%$ and $12.3 \%$ responded only true and wrong that can find ways to get what they want despite some opposition. $48.5 \%$ hits it easy to persist in what is proposed stopping achieve their goals. $67.5 \%$ say it is true that if you try hard enough you can solve difficult problems. Only 40.4\% say they can handle unexpected events effectively.

Keywords: Self-efficacy, Health, universitaries, Incoming students, Nursing, academic performance. 


\section{INTRODUCCIÓN}

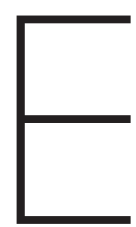

I interés por investigar la autoeficacia en universitarios de nuevo ingreso tiene por objeto identificar y contrastar los hallazgos en estudios similares y extrapolar propuestas de aplicación; mediante intervenciones educativas de apoyo y/o fortalecimiento de la autoeficacia académica tanto teórica como práctica; específicamente para el desempeño exitoso en los escenarios clínicos en donde muchos de los eventos son inesperados y siempre son complejos, especialmente para el alumno que se enfrenta por primera vez a este tipo de ambientes.

La autoeficacia se entiende como la capacidad de las personas de reaccionar con autodominio y habilidades concretas ante situaciones imprevistas o adversas que incluyen las experiencias cotidianas y los estados psicológicos y emocionales que exigen contar con herramientas para un afrontamiento exitoso. ${ }^{1,2,3}$

La especificidad del constructo autoeficacia en universitarios; postulada por la teoría cognitiva social, respecto a que las creencias sobre autoeficacia son específicas de un ámbito de funcionamiento concreto y se diferencia de otros constructos autorreferentes, por ejemplo; autoeficacia académica, autoeficacia estadística, y actitudes hacia la estadística como componentes cognitivoafectivos confirmando que la autoeficacia es diferenciable por campo de dominio o habilidades concretas. $^{4}$

El éxito del estudiante no solo se explica a través de la autoeficacia, sino que es mediado e influyen variables tales como: la dependencia económica, autoestima' bienestar subjetivo y salud general ${ }^{5}$ además de bienestar psicológicob manejo del estrés', entre otros.

El rol que juegan las creencias sobre la autoeficacia en habilidades concretas y el rendimiento académico de universitarios evaluados con la escala de Ralph Schwarzer y Judith Baessler (1996) ${ }^{18}$, mostraron una influencia significativa, positiva y bidireccional entre las creencias de autoeficacia y el rendimiento académico ${ }^{8}$.

La asociación de la autoeficacia percibida y el aprendizaje autorregulado involucran tanto las metas de aprendizaje y la dificultad para la concentración; por lo que es fundamental disponer de estrategias pedagógicas metacognitivas de dominio y de comprensión.?

La relación entre la autoeficacia académica y estrés en estudiantes de posgrado, quienes alcanzan un nivel de autoeficacia percibida alto (73\%) presenta una correlación negativa con el estrés académico, a medida que disminuye la autoeficacia percibida para participar en actividades de interacción y retroalimentación para el aprendizaje, aumenta el estrés en los estudiantes de posgrado?

La correlación entre autoeficacia percibida y ansiedad; mostró que bajas puntuaciones en autoeficacia académica predicen la alta ansiedad: Es posible anticipar que la mejora de las expectativas de autoeficacia aumentaría las probabilidades de disminuir los niveles de ansiedad en los estudiantes. ${ }^{10}$

Usando la Escala de Autoeficacia Percibida Específica de Situaciones Académicas (EAPESA) para evaluar el papel autorregulatorio de la autoeficacia el rendimiento académico y la ansiedad; evidenció que la probabilidad de presentar alta autoeficacia aumenta un 94\% por cada punto que aumenta la nota media, mientras que la probabilidad de presentar éxito académico aumenta un 8\% al incrementarse la autoeficacia académica percibida. ${ }^{11}$

La autoeficacia y la personalidad están estadísticamente relacionadas en sentido positivo, por lo que son predictores y factor protector frente a la aparición de malestar psíquico en personal de enfermería. ${ }^{12}$. De igual manera, la autoeficacia es un mediador entre el bienestar subjetivo y la salud general. ${ }^{5}$

La autoeficacia percibida en universitarios tiene una relación significativa con el bienestar psicológico 
y sus dimensiones: autoaceptación, relaciones positivas con otros, autonomía, dominio del entorno, propósito en la vida y crecimiento personal. ${ }^{6}$

Se ha observado que factores como la dependencia económica, autoestima, trabajo, estado civil y género afectan la autoeficacia en la realización de ejercicio físico como factor protector en el consumo de drogas lo anterior; convalida la pertinencia de contar con programas para fortalecer la autoeficacia y así disminuir el consumo de drogas?. En atletas, la autoeficacia asociada a objetivos y metas personales contrastada con rasgos de ansiedad y rendimiento según género tienen correlación significativa confirmando que la orientación por tareas mejora el rendimiento y mejora las creencias sobre la autoeficacia, en este caso de atletas. ${ }^{13}$

Los perfiles de autoeficacia percibida de hombres y mujeres universitarios en México reportan que las mujeres alcanzan puntuaciones más altas en autoeficacia percibida y con mayor necesidad y posibilidad de ser más autoeficaces. Sugieren que al diseñar intervenciones de mejora de la autoeficacia percibida se considere el factor género. ${ }^{14,75,16}$

Los estudios sobre autoeficacia revisados evidencian la necesidad de incrementar el número de investigaciones en poblaciones universitarias, donde la mayor producción se hace en Norteamérica U.S.A. y Europa, mientras que América Latina tiene un mínimo de investigación sobre el tópico. ${ }^{17}$

Esta investigación de alcance descriptivo, sobre autoeficacia en estudiantes de nuevo ingreso a Enfermería; usando la escala de autoeficacia percibida de Ralph Schwarzer y Judith Baessler (1996) ${ }^{18}$, traducida al español; pretende generar interés por la autoeficacia como categoría a incluir en el análisis del desempeño académico de los alumnos, no solo de nuevo ingreso sino en quienes están en situación de práctica clínica o comunitaria.

\section{MATERIAL Y MÉTODO}

D iseño descriptivo transversal en una población de alumnos de nuevo ingreso a la carrera de enfermería. Se incluyeron a todos los alumnos de primer semestre sumando un total de 342 entrevistados quienes contestaron el formulario el día y la hora de su aplicación. El método de recolección de datos fue una entrevista directa instrumentada utilizando la escala de Dr. Ralph Schwarzer y Dra. Judith Baessler, traducida al español en 1996 por los mismos autores, consta de 10 preguntas en escala tipo Likert que mide el juicio de la capacidad de realizar acciones exitosas. Presenta un patrón de respuesta que va de 1 a 4, donde 1 = incorrecto; 2 = apenas cierto; 3 = más bien cierto y $4=$ cierto; el valor más alto es cuarenta y el más bajo es diez. A mayor puntaje, mayor la autoeficacia percibida. La escala se ha aplicado en población latina y europea, con la obtención de alfa de conbrach de .81 y .93 respectivamente. En este caso con población mexicana calificó con 0.86 .

Los datos se procesaron en SPSS versión 23.0 y se presentan en una tabla con valores porcentuales ordenadas en escala decreciente a partir del îtem "C" Cierto.

\section{RESULTADOS}

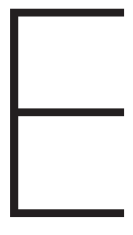
| rango de edad es de 17 a 24 años. El 77.2\% mujeres y el 22.8\% hombres; según estado civil el $66.1 \%$ no tiene pareja y el 37.3\% tiene pareja. El 76\% no tiene empleo y el $71.6 \%$ es dependiente económico. (ver tabla 1)

Respecto a la autoeficacia percibida, al preguntárseles ¿puedes resolver problemas difíciles si te esfuerzas lo suficiente? se observa que el $2.3 \%$ contestaron incorrecto; $7.6 \%$ dijeron que es apenas cierto. El $22.5 \%$ dijeron que es apenas cierto mientras que el 67.5\% expresaron que es cierto, que si se esfuerzan pueden resolver problemas difíciles.

\section{4} MMN $/ W_{4}$ Enf Neurol Vol. 15. No. 3 septiembre - diciembre 2016 
En el rubro ¿puedes resolver la mayoría de los problemas si te esfuerzas lo necesario? el 59.1\% respondió que es cierto, para el 32.7\% fue más bien cierto, el 6.4\% apenas cierto. Sólo 1.8\% dice que lo anterior es incorrecto. Para el 22.5\% es más bien cierto y $67.5 \%$ dice que es cierto, que si se esfuerza lo suficiente puede resolver problemas difíciles.

El 48.5\% acierta a que le es fácil persistir en lo que se propone para lograr sus metas. Un 9.9 dijo que esto es a penas cierto, y un 36.3 \% expresó que es más bien cierto. Sólo un 5.3\% considera que no le es fácil persistir en lo que se propone.

El $42.4 \%$ y el $40.6 \%$ respectivamente indican que es cierto y más bien cierto que gracias a sus cualidades y recursos superan situaciones imprevistas. En cambio, un $14.0 \%$ y $2.9 \%$ responden que es apenas cierto e incorrecto respectivamente.

Para $40.9 \%$ y el 26.3\% contesto Cierto y Más bien cierto que encuentran la manera de obtener lo que quieren a pesar de alguna oposición. En cambio, el 20.2\% y 12.3\% respondió apenas cierto e incorrecto.

Respecto a la confianza para manejar eficazmente acontecimientos inesperados el 4.1\% lo asume como incorrecto; para un $16.1 \%$ es apenas cierto. No obstante, para un 39.5\% es más bien cierto y el 40.4\% dice que puede manejar acontecimientos inesperados eficazmente.

Sobre la capacidad de encontrar alternativas para resolver un problema el 41.2\% señala que es más bien cierto que al tener que hacer frente a un problema, generalmente se les ocurren varias alternativas de cómo resolverlo, en cambio un 15.8\% respondió que es apenas cierto.

Respecto a si se les ocurre qué hacer ante lo difícil el 47.4\% y 36.5\% respondió que es más bien cierto y cierto que generalmente se les ocurre qué hacer. En contraste un $12.6 \%$ y $3.5 \%$ refieren en su respuesta apenas cierto e incorrecto respectivamente.

Cuando se les preguntó si ¿Venga lo que venga eres capaz de manejarlo? el 42.1\% dijo que es más bien cierto y $35.1 \%$ reveló que es cierto. Para un 19.3\% es a penas cierto sólo el 3.5\% indicó que es incorrecto.

Respecto a las habilidades ante dificultades un 44.4\% opina que es más bien cierto que cuando se encuentran en dificultades pueden permanecer tranquilos porque cuentan con habilidades necesarias para tratar ese tipo de situaciones. El 6.4\% carecen de habilidades y para el 20.5\% es apenas cierto que logran tranquilizarse ante situaciones difíciles.

\section{DISCUSIÓN}

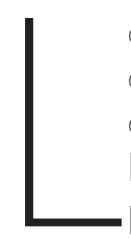

os hallazgos respecto a la disposición al logro de metas en los alumnos de nuevo ingreso coinciden con los de Barraza A, Hernández L.? quien afirma que un nivel alto de autoeficacia - percibida es un indicador de buen renaimiento académico y bajos niveles de estrés. Para el caso de estudiantes de enfermería es deseable monitorear la autoeficacia percibida, específicamente en los campos clínicos; caracterizados por ser generadores de estrés, ansiedad y situaciones inesperadas e imprevistas. Estudios de este tipo coadyuvarán a un proceso de aprendizaje saludable y una mayor probabilidad de éxito académico como lo reportaron Ríos Rísquez M, Sánchez Meca J, Godoy Femández C. ${ }^{2}$

Por el contrario, los casos de bajas puntuaciones en autoeficacia percibida como los observados por García Fernández J M.10 se predicen altos niveles de ansiedad. Lo anterior coincide con Cabanach R. Arias A, Rodríguez C. ${ }^{6}$ quien demuestra que la autoeficacia percibida alta incide sobre el bienestar psicológico, la personalidad y la salud general Sá de Souza L. ${ }^{5}$ siendo al mismo tiempo un predictor, mediador y protector del malestar psíquico, en el caso de las enfermeras en situación de práctica según lo revisado por Ríos Rísquez M, Sánchez Meca J, Godoy Fernández C. ${ }^{12}$

La autoeficacia está asociada a la dependencia económica, autoestima, trabajo, estados civil, bienestar, estrés, ansiedad, salud física y psíquica, rendimiento escolar, bienestar psicológico, salud general, todo en el marco del género. Afectan la Enf Neurol Vol. 15. No. 3 septiembre - diciembre 2016 
autoeficacia en la realización, por ejemplo de ejercicio físico, considerada un factor protector en el consumo de drogas, lo anterior convalida la pertinencia de contar con programas para fortalecer la autoeficacia y así disminuir la exposición a riesgos para para la salud, por ejemplo, las drogas y apoyándose en lo observado por De León A, et al. ${ }^{1}$ fortalecer la autoeficacia en los alumnos ante las dificultades y retos de la práctica clínica.

Cabe mencionar que en este caso no se hace la diferencia en la autoeficacia por género. No obstante, se retoma la propuesta de Aguirre F, Blanco R, Judit M, Rodríguez V. Ornelas M. ${ }^{14}$ quienes compararon la autoeficacia percibida entre hombres y mujeres observando que las mujeres en comparación con los hombres alcanzan altos puntajes de autoeficacia percibida y muestran mayor necesidad y probabilidad de ser más autoeficaces que los varones. Por ende, toda intervención para fortalecer la autoeficacia será diferenciada por género.

No obstante, los buenos resultados tres îtems donde la frecuencia de "Incorrecto" debiera revisarse: a) "permanecer tranquilo en situaciones difíciles" donde 22 casos 6.4\% calificaron en "I". b) persistencia hasta alcanzar metas propuestas se registran 18 casos 5.3\% también ubicados en "l" y el c) "encontrar la manera de obtener lo que quiera, aunque haya oposición" donde 43 casos "20.2\% marco "I". Las tres categorías tienen implicaciones susceptibles de ser atendidas. (VER TABLA 2)

\section{CONCLUSIONES}

a autoeficacia se entiende como la capacidad de las personas de reaccionar con autodominio y habilidades concretas ante situaciones imprevistas o adversas. La escala de Schwrzer \& Baessler ${ }^{18}$ permitió establecer el perfil de los alumnos nuevo ingreso a la carrera de Enfermería, mismos que alcanzaron alto nivel de autoeficacia percibida reflejada en que pueden encontrar la manera de obtener sus propósitos, si se esfuerzan lo necesario y lo suficiente, pueden resolver problemas difíciles, aun cuando encuentren oposiciones.

Destaca que los alumnos de nuevo ingreso cuentan con herramientas de afrontamiento y expresan además que pueden pensar alternativas de solución a sus casos personales. En general, los entrevistados consideran que gracias a sus cualidades y recursos pueden superar situaciones imprevistas. El grupo estudiado reporta además, que les es fácil persistir en lo que se ha propuesto hasta llegar a alcanzar sus metas. Tienen confianza en que podrían manejar eficazmente acontecimientos inesperados.

En conclusión, de acuerdo con la escala Schwarzer, R., Baessler, J, ${ }^{18}$ los alumnos de nuevo ingreso alcanzan altos niveles de autoeficacia percibida ya que consideran, que disponen de herramientas para afrontar situaciones complejas. No obstante, es deseable abordar; desde lo cualitativo; los casos, aunque pocos; en que la autoeficacia no es adecuada toda vez que en enfermería se exige a todos un margen de error mínimo en la práctica clínica, para lo cual se requiere un alto nivel de autoeficacia.

\section{6} MMN/Why Enf Neurol Vol. 15. No. 3 septiembre - diciembre 2016 


\section{REFERENCIAS BIBLIOGRÁFICAS}

1. De León Gómez A, Arrioja Morales G, Antonio López MT, Velasco Parra L, Torres Reyes A, Morales Castillo FA, et al. Conducta de ejercicio y consumo de drogas en estudiantes universitarios. European Journal of Child Development, Education and Psychopathology. (Internet) 2016; 4(1): 5-16. (Consultado el 05/11/2016) Disponible en: http://bit.ly/2fQiULO

2. Cid P, Orellana A, Barriga $\bigcirc$. Validación de la escala de autoeficacia general en Chile. Rev Med Chile (Internet). 2010; 138(5):551-557. (Consultado el 3/10/2016) Disponible en: hittp://bittly/2eE3yqo

3. Ibáñez E, Segura A, Topa C, Autoeficacia específica para la Jubilación. Intenciones de retiro y Satisfacción vital en trabajadores mayores de 40 años Acción Psicológica, (Internet) 2015; 12(1): 41-54, (Consultado el 3/10/2016) Disponible en: http://bit.ly/2feY1bT

4. Blanco Blanco Á. Creencias de la autoeficacia de estudiantes universitarios Un estudio empírico sobre la especificidad del constructo. Revista electrónica de investigación y evaluación educativa. (Internet) 2010; 16(1): 1-28. (Consultado el 05/10/2016) Disponible en: http://bit.ly/2fYbjo/

5. Albuquerque Sá de Souza L, Rosas Torres AR, Alves Barbosa G, Jessé Souza de Lima T. Cunha de Souza LE. Self-efficacy as a mediator of the relationship between subjective well-being and general health of military cadets. Cad. Saúde Pública (Internet) 2014; 30(11): 2309-2319. (Consultado el 19/10/2016) Disponible en: http://bit.ly/2ePN8fa

6. González Cabanach R, Valle Arias A, Freire Rodríquez C, Ferradás Canedo M. Relaciones entre la autoeficacia percibida y el bienestar psicológico en estudiantes universitarios. Revista Mexicana de Psicología (Internet) 2012. 29(1): 40-48. (Consultado el 13/10/2016) Disponible en: http://bit.ly/2eN/IUF

7. Barraza A, Hernández L. Autoeficacia académica y estrés. Análisis de su relación en estudiantes de posgrado. Diálogos educativos. (internet) 2015; 15(30): 21-39. (Consultado el 20/010/2016). Disponible en: http://bit. Iy/2eNkakp

8. José A. Serra Taylor. Autoeficacia y rendimiento académico en estudiantes universitarios. Griot (Internet) 2010; 3(2): $37-45$ (Consultado el 05/10/2016). Disponible en: http://bit.ly/2fAKfO2

9. Pool-Cibrián W. Martínez-Guerrero J. Self-efficacy and Use of Strategies for Self-Regulated Learning in University Students. Revista electrónica de investigación educativa (Internet) 2013; 15(3): 27-36. (Consultado el 13/10/2016). Disponible en: http://bit.ly/2f09QXJ

10. García Fernández JM, Lagos San Martín NG, Gonzálvez Maciá C, Vicent Juan M, Inglés Saura C. ¿Predice la autoeficacia percibida la ansiedad escolar? Estudio con estudiantes chilenos de educación secundaria. International Journal of Developmental and Educational Psychology. (Internet) 2015;1 (1): 193-198. (Consultado el 20/010/2016) Disponible en: http://bit.ly/2dUtPgh
11. García Fernández JM Inglés Saura C, Torregrosa MS, Ruiz Esteban C Díaz Herrero A, Pérez Fernández E, Martínez Monteagudo MC. Propiedades psicométricas de la Escala de Autoeficacia Percibida Específica de Situaciones Académicas en una muestra de estudiantes españoles de Educación Secundaria Obligatoria. European Journal of Education and Psychology (Internet) 2010; 3(1): 2010361-73. (Consultado el 13/10/2016) Disponible en: http://bit.ly/2fkkra3

12. Ríos Rísquez M, Sánchez Meca J, Godoy Fernández C. Personalidad resistente, autoeficacia y estado general de salud en profesionales de Enfermería de cuidados intensivos y urgencias. Psicothema (Internet) 2010:22(4): 600605. (Consultado el 05/17/2016) Disponible en: http://bit.ly/2fEAXju

13. Sari I. Does goal orientation matter for trait anxiety, self-efficacy and performance? An investigation in university athletes. Education (Internet 2015; 136(2): 169-178. (Consultado el 05/11/2016) Disponible en: http://bitt. $1 y / 2 e p 6 k k Q$

14. Aguirre F, Blanco R, Judit M, Rodríguez V, Ornelas M. Autoeficacia percibida en la conducta académica de estudiantes universitarios mexicanos, diferencias entre hombres y mujeres. Formación universitaria (Internet) 2015 8(5): 97-102. (Consultado el 13/10/2016) Disponible en: http://bit.ly/2fIWBFB

15. Blanco Vega H, Ornelas Contreras M, Blanco Ornelas J R, Peinado Pérez J E. Autoeficacia percibida en conductas académicas en universitarios Un estudio respecto de alumnos de educación física. Revista Mexicana de Investigación Educativa (Internet) 17(54): 779-791. (Consultado el 05/10/2016). Disponible en: http://bitily/2fY6CjO

16. Ornelas M. Blanco H. Gastélum G, Chávez A. Autoeficacia percibida en la conducta académica en estudiantes universitarias. Formación universitaria (Internet) 5(2): 17-26. (Consultado el 05/10/2016). Disponible en: http://bit. ly/2eQrkpp

17. Reyes K, Hernández M. Análisis crítico de los estudios que exploran la autoeficacia y el bienestar vinculados al comportamiento saludable. Journa Behavior (Internet) 2011: 3(2): 5-24. (Consultado el 05/11/2016). Disponible en: http://bit.ly/2endy8z

18. Schwarzer, R., Baessler, J. Evaluación de la autoeficacia: adaptación española de la Escala de Autoeficacia General. Ansiedad y estrés. (Internet) 1996 2(1): 1-8. (Consultado el 13/10/2015) Disponible en: hitp://bit.ly/2e5ihd/H 
Tabla 1. Distribución según edad y género de los alumnos de nuevo ingreso a la licenciatura en enfermería entrevistados sobre autoeficacia. 2016

\begin{tabular}{c:c:c:c} 
EDAD & MASCULINO & FEMENINO & TOTAL \\
$15-19$ & 172 & 34 & 206 \\
$20-24$ & 76 & 38 & 114 \\
$25-29$ & 8 & 4 & 12 \\
$30-34$ & 3 & 1 & 4 \\
$35-39$ & 2 & 1 & 3 \\
$40-+$ & 3 & 0 & 3 \\
\hdashline TOTAL & 264 & 78 & 342 \\
\hline
\end{tabular}

Fuente: Datos elaborados por: Nájera Vuelvas J, Pacheco Arce AL, Rodríguez Becerra C, Téllez Ortiz II, Sánchez Estrada T. ENEO-UNAM 2016

Tabla 2. Autoeficacia percibida en alumnos de nuevo ingreso a la licenciatura en enfermería. 2016

\begin{tabular}{|c|c|c|c|}
\hline 1 & $A C$ & MBC & C \\
\hline Incorrecto & Apenas Cierto & Más Bien Cierto & Cierto \\
\hline
\end{tabular}

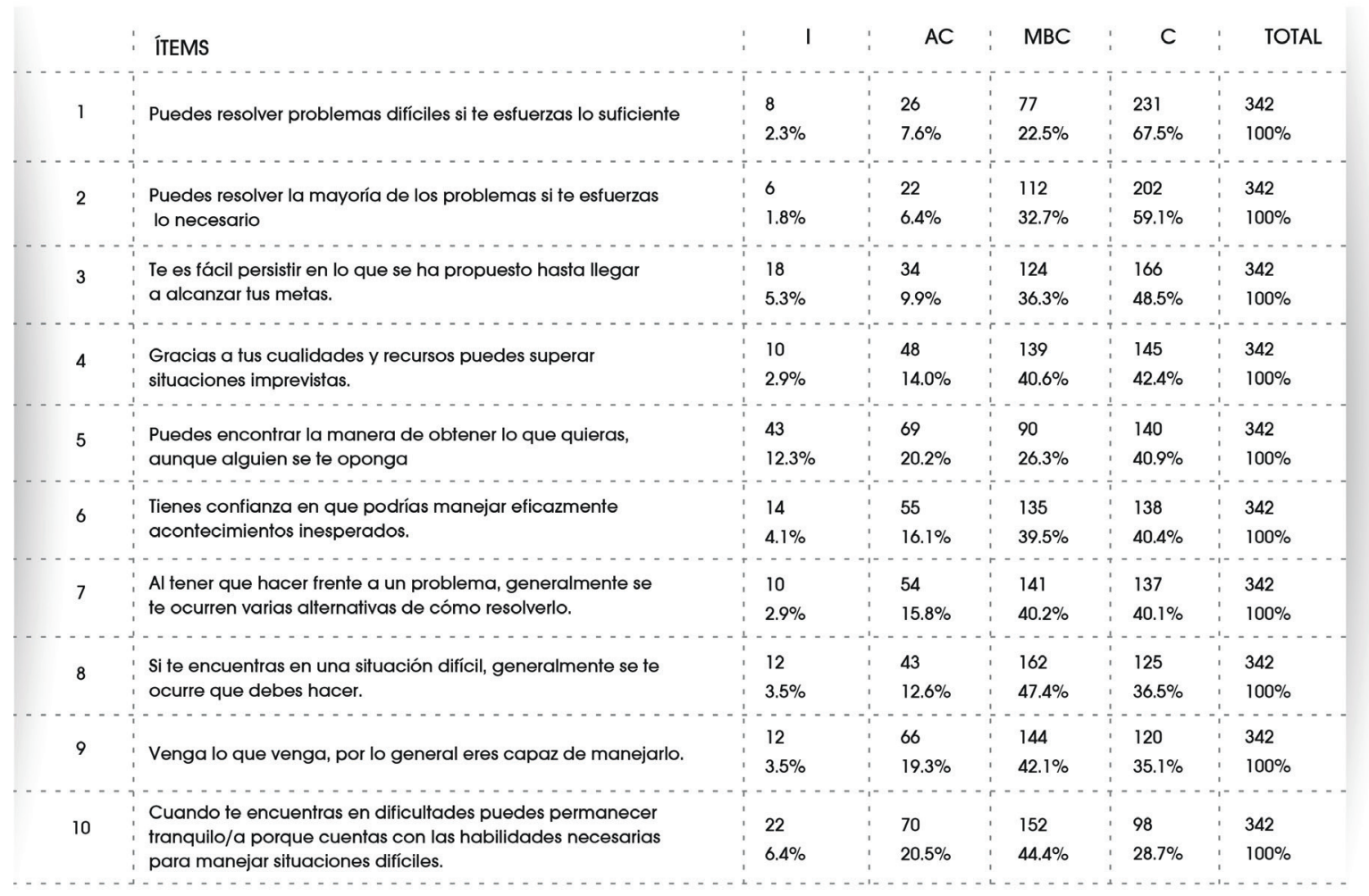

Fuente: Datos elaborados por: Nájera Vuelvas J, Pacheco Arce AL, Rodríguez Becerra C, Téllez Ortiz II, Sánchez Estrada T. ENEO-UNAM 2016 